\title{
Chapter 19 \\ Origin of Female/Male Gender as Deduced by the Mating-Type Loci of the Colonial Volvocalean Greens
}

\author{
Hisayoshi Nozaki
}

\begin{abstract}
Colonial Volvocales (green algae) are a model lineage for the study of the evolution of sexual reproduction because isogamy, anisogamy, and oogamy are recognized within the closely related group, and several mating type (sex)-specific genes were identified in the closely related unicellular Chlamydomonas reinhardtii during the past century. In 2006, we first identified a sex-specific gene within the colonial Volvocales using the anisogamous colonial volvocalean alga Pleodorina starrii, namely, a male-specific gene called "OTOKOGI," which is a homologue of the minus mating type-determining gene MID of the isogamous $C$. reinhardtii. Thus, it was speculated that the derived or minus mating type of $C$. reinhardtii is homologous to the male in the anisogamous/oogamous members of the colonial Volvocales. The discovery of the male-specific gene facilitated comparative studies of the mating-type locus (MT) (primitive sex chromosomal region) because it must be localized in $M T$. Recently, our international research group determined the genome sequence of $M T$ in the oogamous Volvox carteri. V. carteri MT shows remarkable expansion and divergence relative to that from $C$. reinhardtii. Five new female-limited "HIBOTAN" genes and ten male-limited genes (including "OTOKOGI") were identified in V. carteri MT. These observations suggest that the origins of femaleness and maleness are principally affected by the evolution of $M T$, which has undergone a remarkable expansion and gain of new male- and femalelimited genes. Our recent results regarding the evolution of the volvocalean $M T$ gene $M A T 3 / R B$ are also discussed in relationship to the evolution of male-female sexual dimorphism.
\end{abstract}

Keywords Gametes $\bullet$ Male-female sexual dimorphism $\bullet$ Mating-type locus $\bullet$ Sexspecific gene $\bullet$ Volvocales

H. Nozaki $(\bowtie)$

Department of Biological Sciences, Graduate School of Science, University of Tokyo, Hongo, Bunkyo-ku, Tokyo 113-0033, Japan

e-mail: nozaki@biol.s.u-tokyo.ac.jp 


\subsection{Introduction}

There is a great deal of interest in sexual reproduction because we are the progeny of our parents who produced sperm and immotile eggs (oogamy), which differ markedly in size and motility. In contrast, primitive organisms sometimes exhibit isogamy (equally sized gametes); in this case, we cannot distinguish between males and females. Therefore, evolutionary transition from isogamy to anisogamy (small and large motile gametes) to oogamy (Bold and Wynne 1985), or the origin of male and female sexes, is one of the most interesting issues in biological research. Although such male-female sexual dimorphism has arisen repeatedly in the evolution of eukaryotes (Kirk 2006), no molecular genetic evidence had been reported for the evolutionary link between male and female and mating types of isogamy until our recent study (Nozaki et al. 2006a), possibly because animals and land plants have no extant isogamous relatives (Rokas et al. 2005; Laurin-Lemay et al. 2012).

Colonial volvocalean algae represent a useful model lineage for the study of such an evolutionary link because isogamy, anisogamy, and oogamy can be recognized within the closely related organisms (Nozaki et al. 2000; Herron et al. 2009), and several mating type-specific genes had been identified in the related unicellular model species, Chlamydomonas reinhardtii (Ferris and Goodenough 1994, 1997). This review focuses on studies of sexual reproduction of the colonial Volvocales as well as the related $C$. reinhardtii.

\subsection{Sexual Reproduction in the Colonial Volvocales}

Figure 19.1 presents a summary of the vegetative organization and modes of sexual reproduction in volvocine algae. It is interesting that, in this lineage, the primitive colonial organization also has a primitive type of sexual reproduction (Nozaki and Ito 1994).

In the colonial volvocalean Gonium pectorale, vegetative spheroids are flattened with 8 or 16 cells, and sexual reproduction is isogamous with no differentiation between female and male gametes. Both the two conjugating isogametes bear a mating papilla, which is called the bilateral mating papilla (Nozaki 1984, 1996; Mogi et al. 2012) (Figs. 19.1, 19.2). Plasmogamy is initiated by the union of the tips of the mating papillae from mating-type plus and minus gametes.

In Eudorina elegans, vegetative spheroids are spheroidal with 16 or 32 cells, and sexual reproduction is anisogamous with flagellate female and male gametes. In sexual reproduction, bundles of male gametes called "sperm packets" are formed. Sperm packets swim to a female spheroid and dissociate into individual male gametes that penetrate the female for gametic union (Goldstein 1964; Nozaki 1983, 1996) (Fig. 19.3).

Volvox carteri is oogamous and has more than 500 cells in a spheroid. When inducing sexual reproduction, dwarf male and special female sexual spheroids are formed. The male spheroid produces sperm packets that swim to the female spheroid, which contains a number of eggs (Nozaki 1988) (Fig. 19.4). V. carteri shows prominent male-female differentiation (Kirk 1998; Nozaki 1996). 

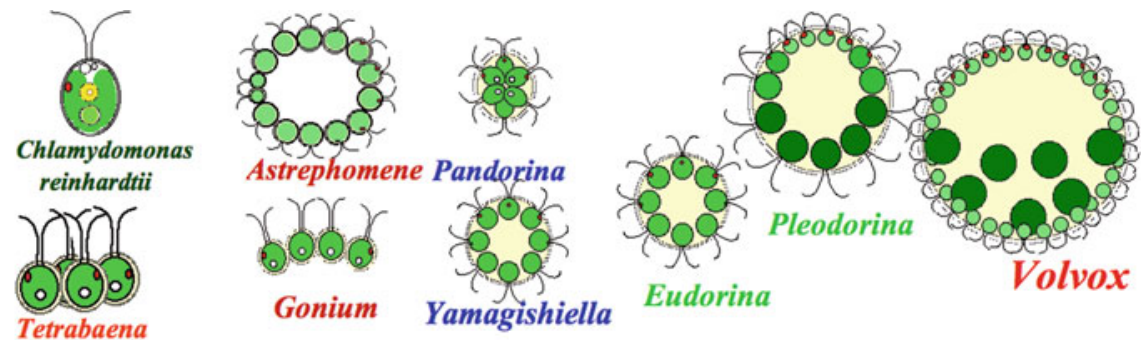

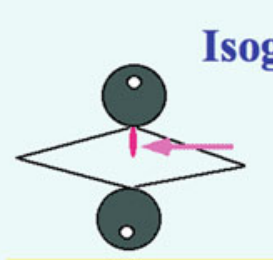

unilateral mating mating papilla

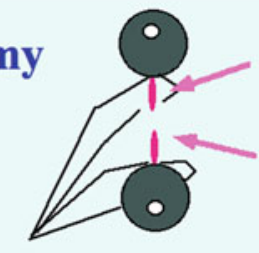

bilateral mating mating papilla

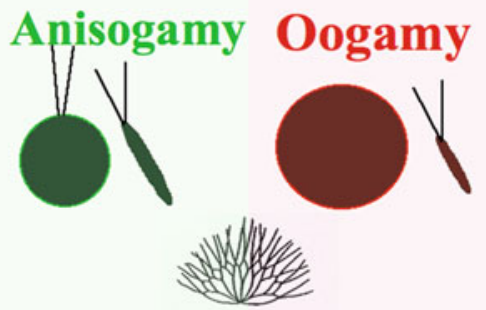

sperm packets

Fig. 19.1 Diagrams of vegetative organization and sexual reproduction in various members of the colonial Volvocales and Chlamydomonas reinhardtii. (Based on Nozaki and Ito 1994)

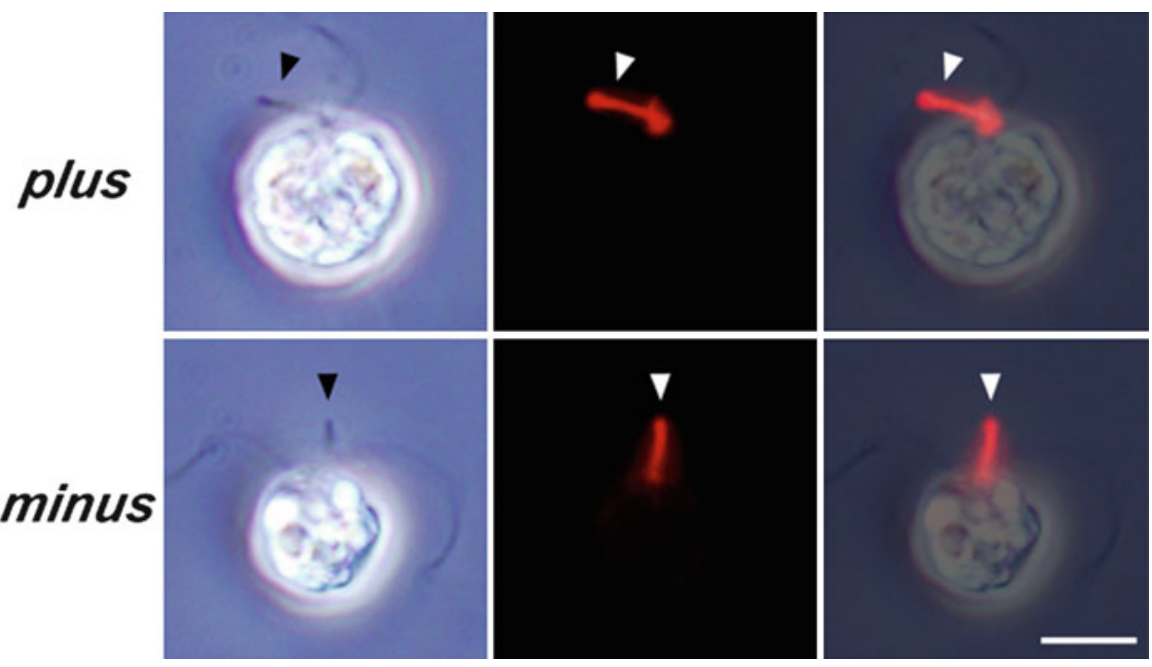

Fig. 19.2 Light microscopy of Gonium pectorale isogametes induced in separate mating type plus (plus) and mating type minus (minus) cultures. All adjacent panels show the same cell. Bar $10 \mu \mathrm{m}$. Phase-contrast (left) and immunofluorescence (actin) (middle) images are merged in the right panels (merge). Note naked gametes of both mating types bearing a tubular mating structure (arrowheads). Note the accumulation of actin (red) in the mating structure. (From Mogi et al. 2012; reproduced by permission of John Wiley and Sons, License No. 3079280348215) 


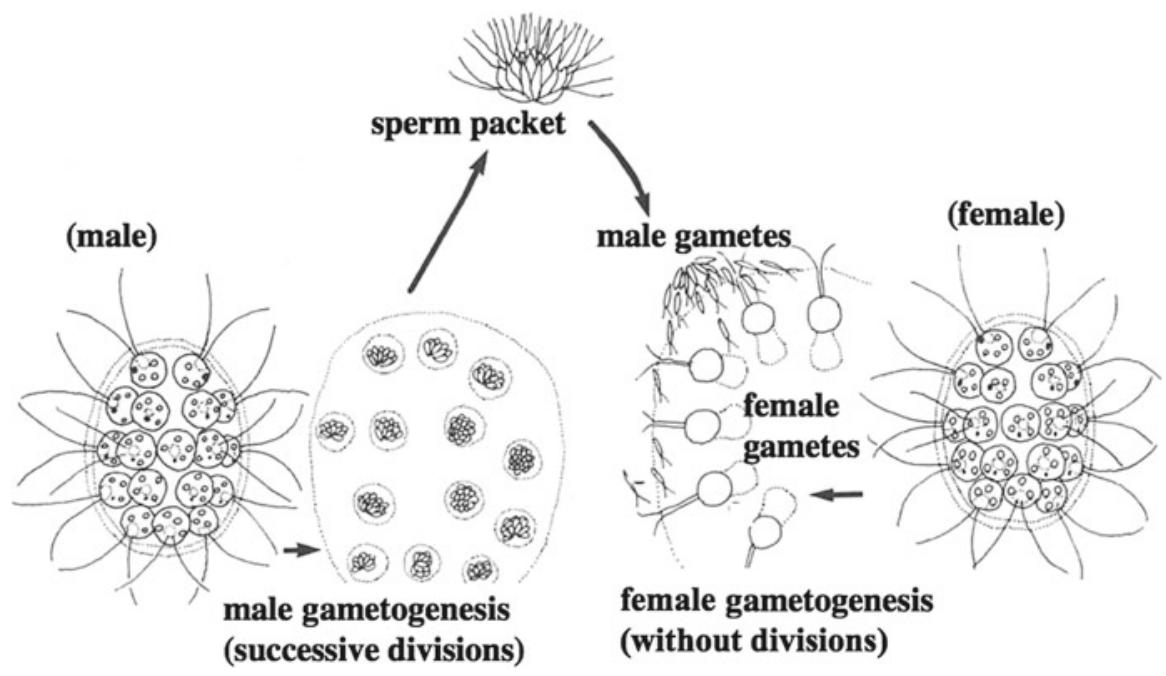

Fig. 19.3 Diagrams of anisogamous sexual reproduction in Eudorina elegans. (Based on Nozaki 1983)

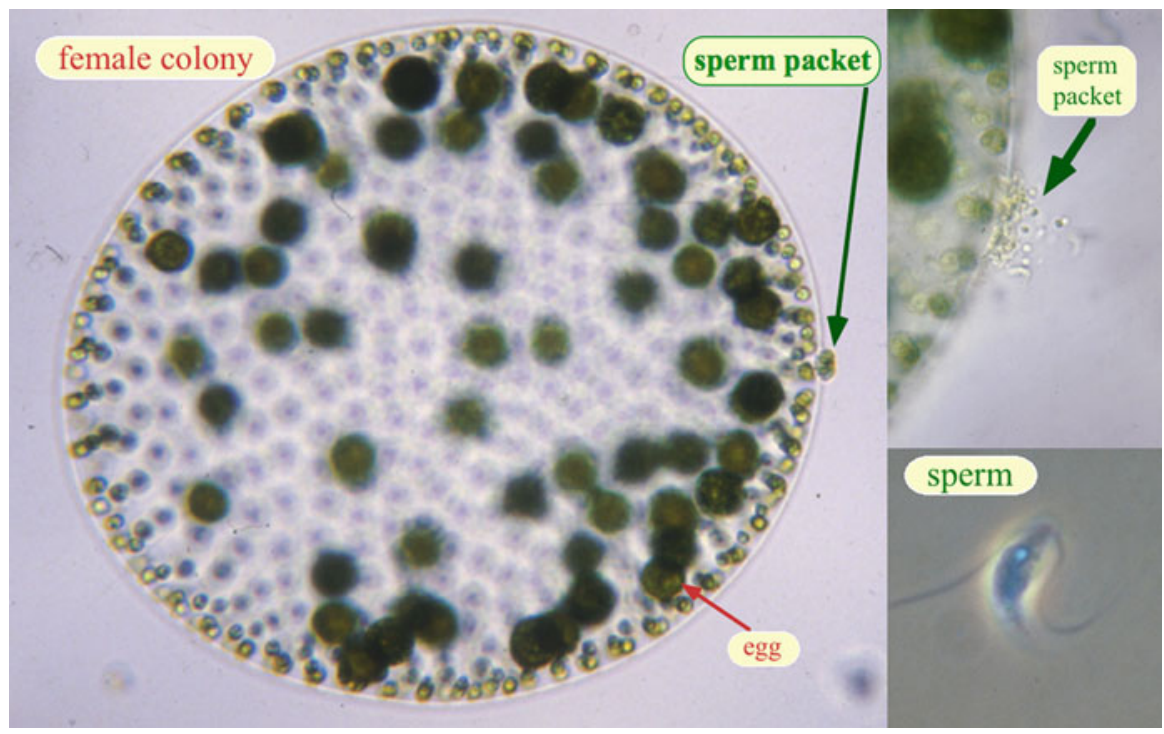

Fig. 19.4 Oogamous sexual reproduction of the multicellular green alga Volvox carteri f. kawasakiensis. Female spheroids and male spheroids with sperm packets develop in female and male strains, respectively, when sexually induced. The sperm packet attaching to the female spheroid containing many eggs (left panel). Sperm packet (arrow) dissociates into individual sperm that penetrate the female spheroid (right upper panel). Sperm (right lower panel). (Original photographs of the strains used by Nozaki 1988) 


\subsection{Determining the Evolutionary Process of Sexual Reproduction and Tracing Gender-Specific Genes of the Colonial Volvocales}

To determine the evolutionary process of the sexual reproduction characteristics in colonial Volvocales, detailed and robust phylogenetic relationships of the members are needed. Therefore, we carried out cladistic analysis of morphological characteristics of vegetative and reproductive phases (Nozaki and Ito 1994; Nozaki et al. 1996) and multigene phylogeny of plastid protein-coding genes (Nozaki et al. 2000; Nozaki 2003). Based on our phylogenetic analyses of morphological and multigene sequence data, a possible evolutionary scenario of sexual reproduction could be deduced. In relationship to the increase in spheroid cell number, female and male genders evolved with the formation of sperm packets from isogamy with bilateral mating papillae (Fig. 19.5). Therefore, it was of interest to determine the genetic basis underlying this evolution of sex. However, mating type-specific genes had not

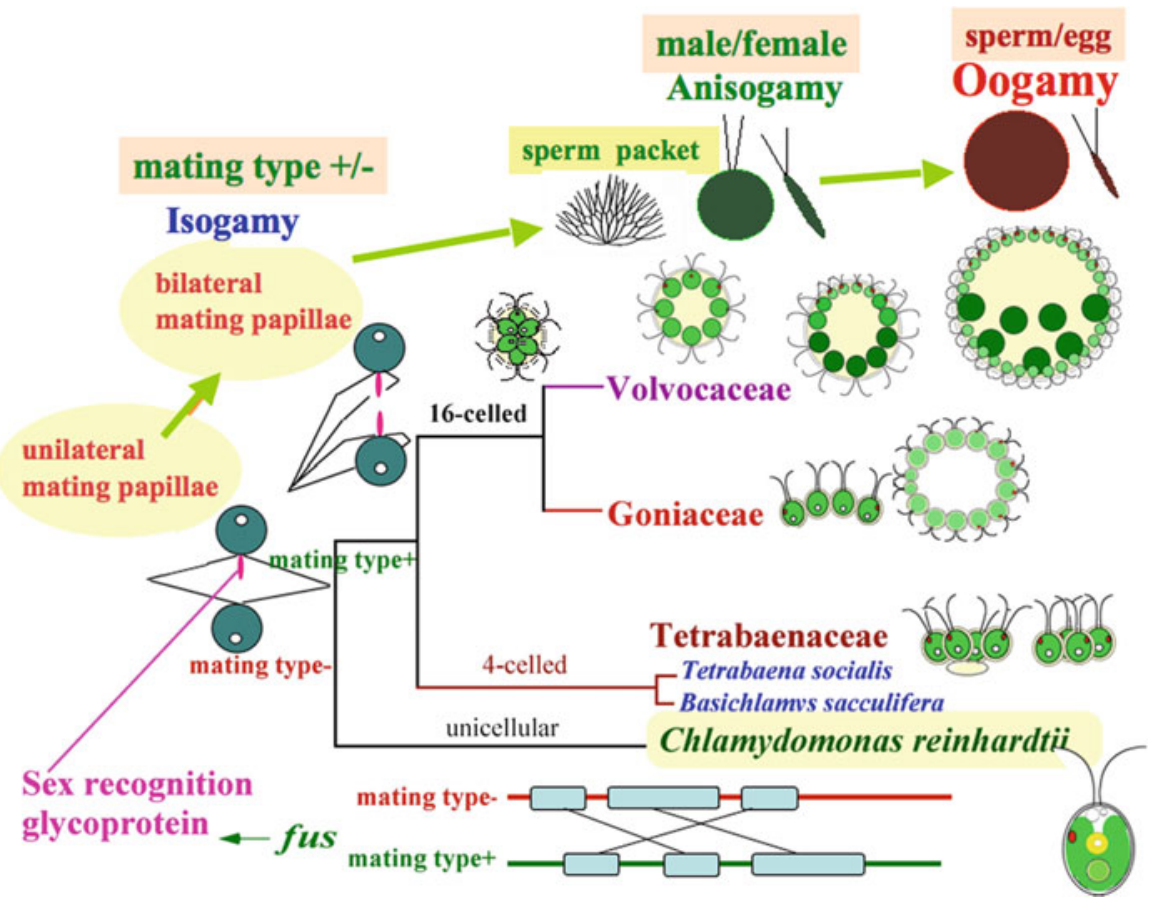

Fig. 19.5 Schematic representation of phylogenetic relationships within the colonial Volvocales as inferred from morphological and multigene sequence data (Nozaki and Ito 1994; Nozaki et al. 2000; Nozaki 2003). A possible evolutionary scenario of sexual reproduction is also shown 
been identified within colonial Volvocales, despite studies of the mating type-specific genes, including the sex-determining minus dominance $(M I D)$ gene in the close relative C. reinhardtii (Ferris and Goodenough 1994, 1997).

There are two possible explanations for difficulties in identifying mating type-specific genes from colonial Volvocales. The first is the rapid evolution of the sex-related genes. Ferris et al. (1997) could not isolate MID homologues from G. pectorale and V. carteri based on Southern blotting, and they discussed the rapid evolution of sex-related genes. The second is loss of fertility or sexual activity during long-term maintenance of culture strains (Coleman 1975). Strains obtained from the culture collections that have been maintained in living culture for more than 10 years cannot generally be induced to reproduce sexually to study sexual reproduction, especially in anisogamous and oogamous species. Therefore, we used newly designed degenerate polymerase chain reaction (PCR) primers and new strains of the anisogamous volvocalean Pleodorina starrii to identify genderspecific genes. P. starrii was described by Nozaki et al. (2006a) based on samples collected in Japanese lakes in 2000-2001, and it is heterothallic with sperm packet formation occurring even within the male strain (Nozaki et al. 2006a, b).

\subsection{Problems Regarding the Evolutionary Link Between Isogametic Mating Types and Male-Female Differentiation}

In volvocine algae, sex is determined by a single mating-type locus $(M T)$ with two haplotypes that specify sexual differentiation. Although $M T$ segregates as a single Mendelian trait, it is a complex genomic region containing both shared and sexlimited genes that are rearranged with respect to each other and do not undergo meiotic recombination (Umen 2011). Sexual reproduction of the unicellular volvocalean $C$. reinhardtii is isogametic, with plus and minus mating types. The minus mating type of this species represents a "dominant sex" because occasionally produced diploid cells exhibit the minus mating phenotype (Ebersold 1967); when the minus mating type-determining gene MID is lacking, the phenotype of the sex changes from minus to plus, forming a fertilization tubule (Goodenough et al. 1982). Therefore, mating type minus in C. reinhardtii can be considered a "derived type of sex." However, the evolutionary link between isogamous mating type plus/minus and female-male differentiation cannot be deduced, even based on comparison of modes of uniparental inheritance of the organelle genomes. In C. reinhardtii, the plastid genome is inherited from the plus mating type parent, whereas the mitochondrial genome is inherited from the minus parent (Boynton et al. 1987). In contrast, both the plastid and mitochondrial genomes are inherited from the female parent in the oogamous alga V. carteri (Adams et al. 1990). Thus, homologues of some C. reinhardtii mating type-specific genes were needed to be identified in the anisogamous or oogamous members of colonial Volvocales. 


\subsection{Discovery of the Male-Specific Gene "OTOKOGI"}

The male-specific gene isolated from $P$. starrii is a homologue of the MID gene of C. reinhardtii (Nozaki et al. 2006a). The MID gene is located in the rearranged (R) domain of the minus MT or primitive sex chromosome in $C$. reinhardtii (Ferris and Goodenough 1994). The $\mathrm{R}$ domain in the sex chromosome is different between plus and minus mating types. To isolate the MID gene in $P$. starrii, new degenerate primers were designed, and RNA was extracted from $P$. starrii male cultures that produced abundant male gametes in nitrogen-deficient medium (Nozaki et al. 2006a).

The new male-specific gene (PlestMID) is called "OTOKOGI," meaning "manliness" or "chivalry" in Japanese (Nozaki et al. 2006a; Nozaki 2008). Figure 19.6 shows the amino acid alignment of the Pleodorina MID gene, i.e., "OTOKOGI," and two Chlamydomonas MID genes. These three genes have essentially the same molecular structure with an RWP-RK domain (Fig. 19.6). Southern blotting analysis indicated that both "OTOKOGI" and its pseudogene are present in the nuclear genome of the male strain but lacking in the female strain (Nozaki et al. 2006a). Genomic PCR of six Pleodorina strains also demonstrated that "OTOKOGI" and its pseudogene are present only in male strains (Nozaki et al. 2006a). Using antibody staining, "OTOKOGI" protein was shown to be accumulated in the male gametic nuclei (Nozaki et al. 2006a) (Fig. 19.7). Therefore, the Pleodorina "OTOKOGI" gene may participate in differentiation of male gametes, and thus the dominant or minus mating type of the isogamous $C$. reinhardtii is homologous to the male in the anisogamous volvocalean Pleodorina. In Volvocales, the derived type of sex evolved to male, whereas the basic type of sex evolved to female.

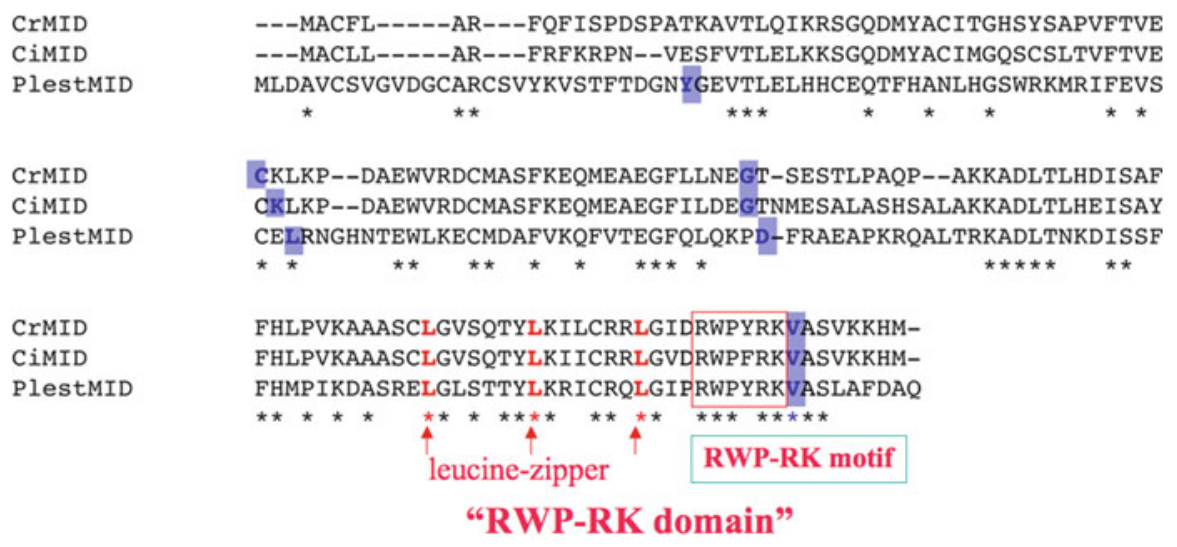

Fig. 19.6 Alignment of the sequences of male-specific "OTOKOGI" and two Chlamydomonas MID proteins (CrMID and CiMID). Blue shading indicates intron locations. (From Nozaki et al. 2006a; reproduced by permission of Elsevier Ltd., License No. 3079280808611) 


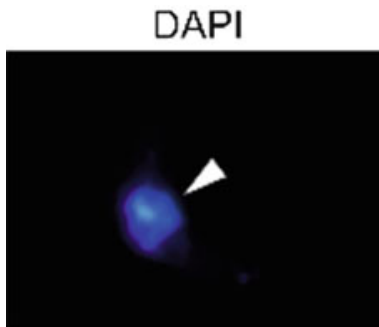

Immunofluorescence
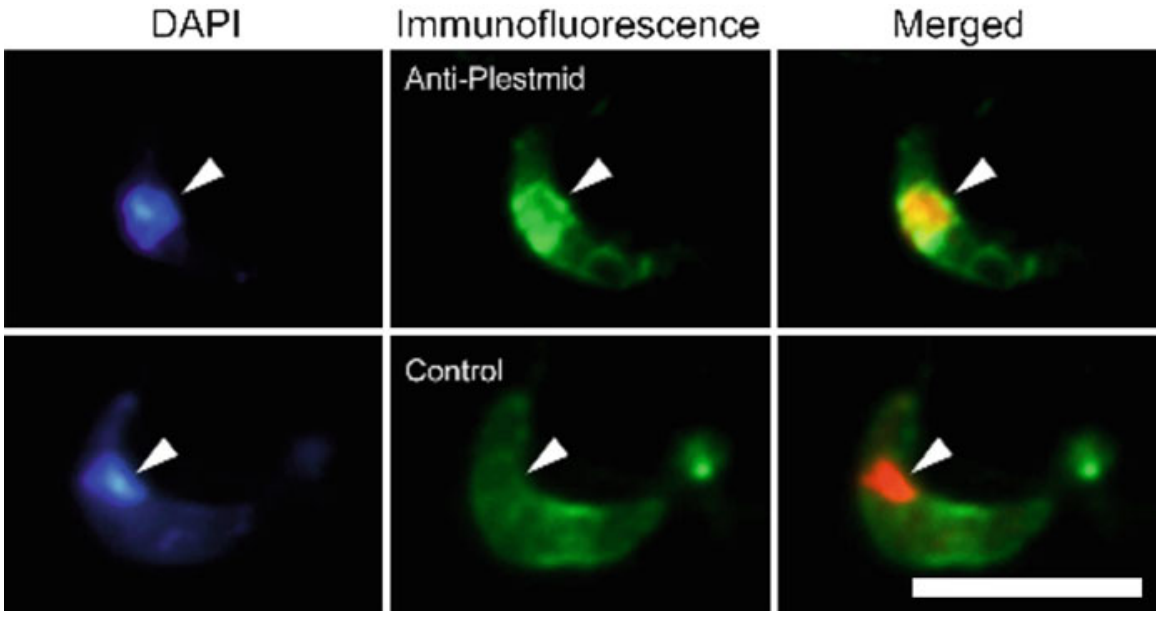

Fig. 19.7 Visualization of male-specific "OTOKOGI" protein in male gamete after release from sperm packets. "OTOKOGI" expression is obvious in nucleus (arrowheads in DAPI images). Specimens were double stained with 4',6-diamidino-2-phenylindole (DAPI) and anti-"OTOKOGI" antibodies. All panels show identical cells. DAPI (pseudo-colored) and immunofluorescence images are merged (right panels). Bar $5 \mu \mathrm{m}$. (From Nozaki et al. 2006a,b; reproduced by permission of Elsevier Ltd., License No. 3079280808611)

\subsection{Female-Limited "HIBOTAN" Genes Resolved in the Volvox carteri Genome}

Even though the evolutionary link between male-female differentiation and isogametic mating types was determined (Nozaki et al. 2006a), genes contributing to the evolution of femaleness and maleness from isogametic mating types remained unclear. In colonial Volvocales, the female sex evolved from the original type of sex (plus mating type) in isogamy (Nozaki et al. 2006a), and female-specific genes are lacking or rare in higher animals and land plants. Thus, the female-specific gene "HIBOTAN" may be lacking in the evolution of femaleness of colonial Volvocales. Genomic data regarding $M T$ or the sex chromosomal region of the anisogamous or oogamous members were needed.

In 2010, we published a paper on the genome analyses of the MT of $V$. carteri that exhibits the production of eggs and sperm (Ferris et al. 2010). This publication was based on co-research between the United States (USA) and Japan, which originally started in 2005 when Dr. Patrick Ferris came to Japan and became a guest researcher at the Graduate School of Science, University of Tokyo. The Japanese team contributed to cloning of the V. carteri "OTOKOGI" gene and construction of a male $V$. carteri BAC library.

The V. carteri MT shows remarkable expansion and divergence relative to that from $C$. reinhardtii, which has equal-sized gametes, and is about five times larger and contains more genes than the $C$. reinhardtii $M T$. Our transcriptome analysis 


\section{Oogamy "Volvox"}

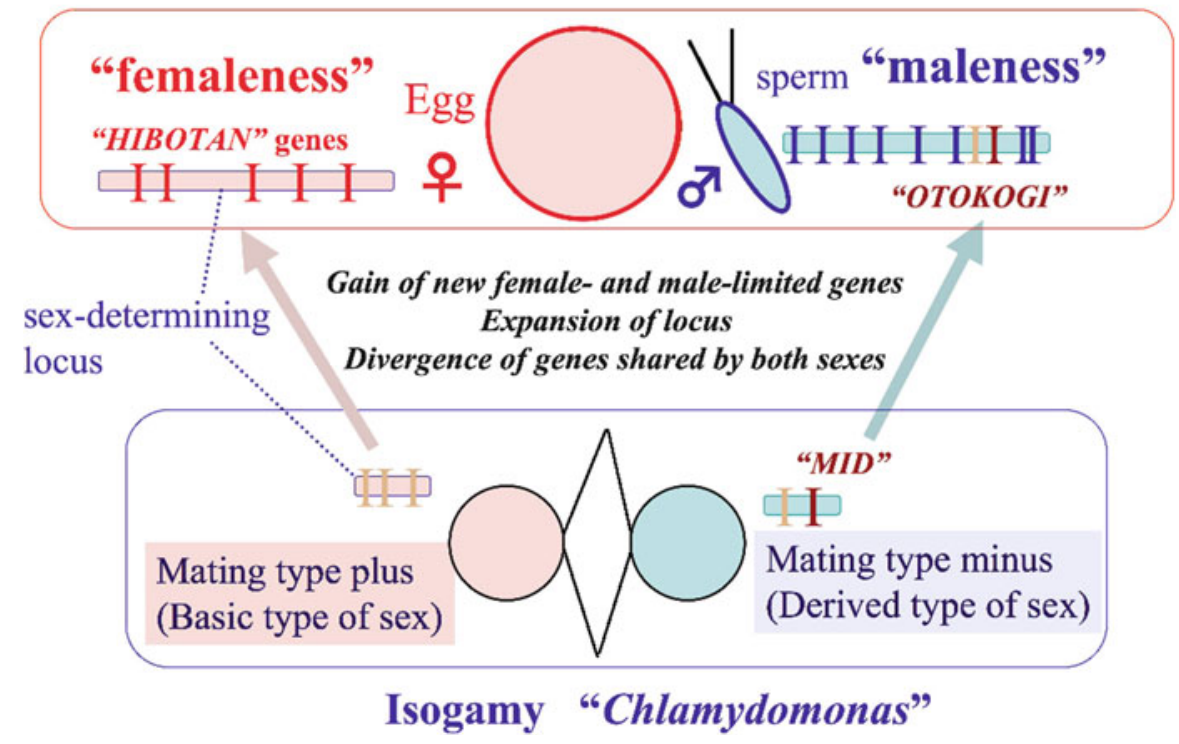

Fig. 19.8 Diagram of evolution of oogamy from isogamy and mating type (sex-determining) loci in the volvocalean algae. (Based on Nozaki et al. 2006a and Ferris et al. 2010)

using next-generation sequencing identified five new female-limited "HIBOTAN" genes and ten male-limited genes, including "OTOKOGI," in the MT locus of $V$. carteri (Fig. 19.8). None of the five "HIBOTAN" genes was identified in the C. reinhardtii $M T$ (Ferris et al. 2010). Thus, the origins of femaleness and maleness are principally affected by the evolution of the $M T$ or sex chromosome that has undergone remarkable expansion and gain of new male- and female-limited genes. However, our study (Ferris et al. 2010) was based on the comparison of only two evolutionary extremes, the isogamous unicellular Chlamydomonas and the oogamous multicellular Volvox (Fig. 19.5). Thus, genome information on the MT from intermediate colonial Volvocales is needed to determine the evolutionary significance of expansion of the MT as well as the male- and female-limited genes newly identified in V. carteri (Charlesworth and Charlesworth 2010).

\subsection{Origin of the Gender-Based Divergence of the Mating Locus Gene MAT3 from Oogamous Volvox carteri}

Our recent comparative study of $M T$ from oogamous $V$. carteri, with $M T$ from an isogamous species, $C$. reinhardtii, revealed major differences in the size and sexbased differentiation of $M T$ genes (Ferris et al. 2010). V. carteri MT was found to show a much higher degree of sex-based differentiation in its shared genes (those 


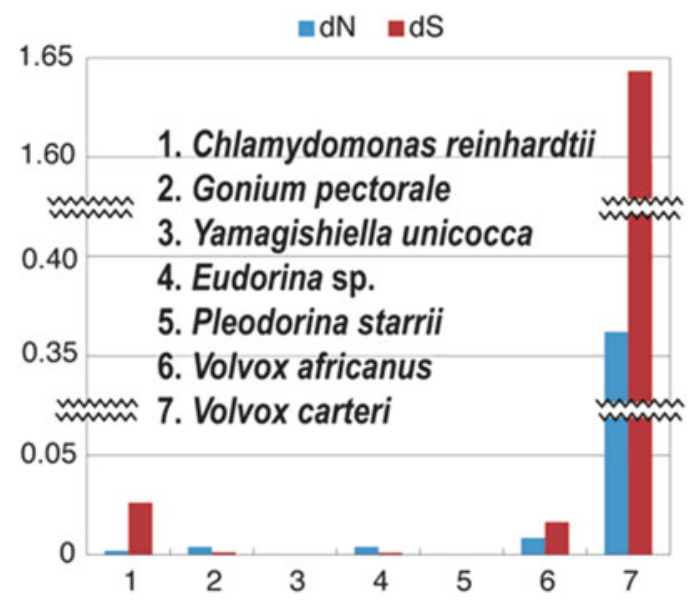

Fig. 19.9 Gender-based divergence of mating-type locus MAT3 genes from the colonial Volvocales and Chlamydomonas reinhardtii. Bar graph depicts $\mathrm{dN}$ (number of substitutions per nonsynonymous site) and dS (number of substitutions per synonymous site) between MAT3 alleles from each of the two mating types or sexes. (From Hiraide et al. 2013; reproduced by permission of Oxford University Press, License Number 3079271441882)

with an allele in both mating haplotypes or sexes). Shared genes that have become masculinized and feminized in sequence or expression, as occurred in V. carteri, are candidates for contributing to male-female sexual dimorphism. However, there had been no investigations of $M T$ genes in the context of the isogamy to anisogamy/oogamy transition in volvocine algae other than the previously described comparison of C. reinhardtii and V. carteri (Ferris et al. 2010; Charlesworth and Charlesworth 2010).

One candidate regulator of gamete size is the mating locus gene MAT3, which encodes a homologue of the retinoblastoma (RB) tumor suppressor protein. In $C$. reinhardtii, MAT3 is closely linked to $M T$ and regulates cell size and cell-cycle progression (Umen and Goodenough 2001; Fang et al. 2006). In contrast to C. reinhardtii, in which the minus and plus MAT3 alleles are nearly identical and function interchangeably (Umen and Goodenough 2001; Merchant et al. 2007), high degrees of male-female sequence differentiation and sex-regulated alternative splicing were observed for V. carteri MAT3 (Ferris et al. 2010). These observations led Ferris et al. (2010) to suggest that MAT3 homologues may be related to control of gamete size in colonial volvocine algae, as predicted earlier by the gamete size regulator recruitment model for the evolution of anisogamy/oogamy from isogamous mating types (Charlesworth 1978). Thus, Hiraide et al. (2013) sequenced the full-length coding regions of MAT3 from plus and minus mating types of isogamous Gonium pectorale and Yamagishiella unicocca, from males and females of anisogamous Eudorina sp. and Pleodorina starrii, and from males and females of oogamous Volvox africanus. In contrast to $V$. carteri, MAT3 homologues from the five colonial species examined had almost identical nucleotide sequences between the two sexes (Fig. 19.9). Our phylogenetic analysis of MAT3 sequences suggested that the extensive MAT3 


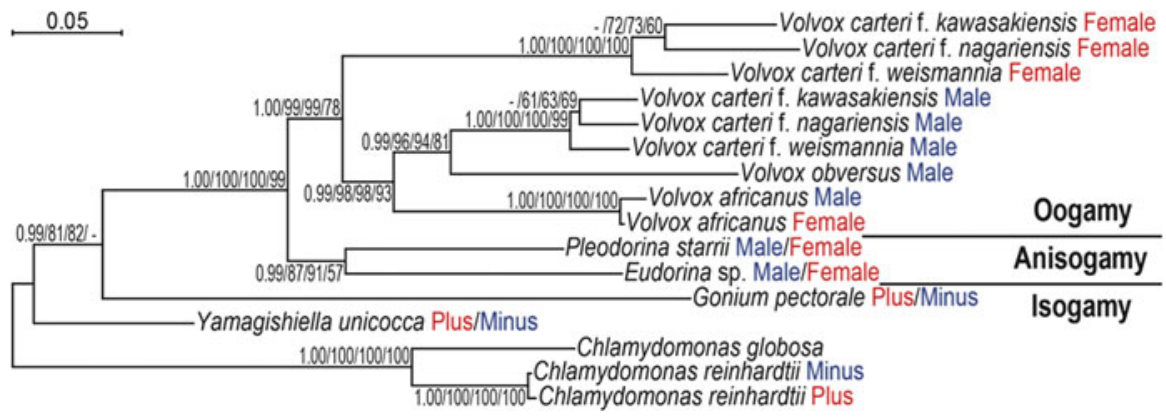

Fig. 19.10 Phylogeny of deduced amino acids of mating-type locus MAT3 genes from colonial Volvocales and Chlamydomonas. Branch labels indicate, from left to right: posterior probabilities $(\geq 0.90)$ from Bayesian inference/bootstrap values $(\geq 50 \%)$ obtained using 1,000 replicates with RAxML/Bootstrap values ( $\geq 50 \%$ ) using 1,000 replicates with maximum parsimony. (From Hiraide et al. 2013; reproduced by permission of Oxford University Press, License Number 3079271441882)

divergence in the $V$. carteri lineage may have occurred recently in the ancestor of the three $V$. carteri forms after their divergence from the anisogamous lineage containing P. starrii and Eudorina sp. (Fig. 19.10). These observations suggest the roles of genetic determinants other than or in addition to MAT3 in the evolution of anisogamy in colonial volvocalean algae.

\subsection{Conclusions}

The considerable expansion of oogamous $V$. carteri $M T$ is based mostly on the increase in the noncoding DNA region, but $V$. carteri $M T$ contains more coding regions or genes than $C$. reinhardtii $M T$ (Ferris et al. 2010). Similar expansion of noncoding DNA regions was recently recognized in the mitochondrial and plastid genomes from colonial Volvocales (Smith and Lee 2009, 2010; Hamaji et al. 2013; Smith et al. 2013). Smith et al. (2013) demonstrated that ratios of noncoding DNA regions in the mitochondrial and plastid genomes increase in relationship to the increase in spheroid cell number (from unicellular Chlamydomonas to Gonium to Pleodorina to Volvox) (see Fig. 19.1). This increase may be explained by the mutational hazard hypothesis (Lynch 2007), which argues that genome expansion is a product of a low effective population size $(\mathrm{Ne})$, which results in increased random genetic drift, or a low mutation rate $(\mu)$, which reduces the burden of harboring excess DNA. Therefore, a gradual increase in $M T$ size as a function of the increase in spheroid cell number, which may result in a decrease in Ne, may also be considered within colonial Volvocales. However, the origins and evolution of the many gender-specific genes found in V. carteri MT remain unexplained on the basis of the present limited data from colonial volvocalean $M T$. 
Acknowledgments This work was supported by the MEXT/JSPS KAKENHI (grant numbers 24112707 and 24247042 to H.N.).

Open Access: This article is distributed under the terms of the Creative Commons Attribution Noncommercial License which permits any noncommercial use, distribution, and reproduction in any medium, provided the original author(s) and source are credited.

\section{References}

Adams CR, Stamer KA, Miller JK et al (1990) Patterns of organellar and nuclear inheritance among progeny of two geographically isolated strains of Volvox carteri. Curr Genet 18:141-153

Bold HC, Wynne MJ (1985) Introduction to the algae: structure and reproduction, 2nd edn. Prentice-Hall, Englewood Cliffs

Boynton JE, Harris EH, Burkhart BD et al (1987) Transmission of mitochondrial and chloroplast genomes in crosses of Chlamydomonas. Proc Natl Acad Sci USA 84:2391-2395

Charlesworth B (1978) The population genetics of anisogamy. J Theor Biol 73:347-357

Charlesworth D, Charlesworth B (2010) Evolutionary biology: the origins of two sexes. Curr Biol 20:R519-R521

Coleman AW (1975) Long-term maintenance of fertile algal clones: experience with Pandorina (Chlorophyceae). J Phycol 11:282-286

Ebersold WT (1967) Chlamydomonas reinhardi: heterozygous diploid strains. Science 157:447-449

Fang S-C, de los Reyes C, Umen JG (2006) Cell size checkpoint control by the retinoblastoma tumor suppressor pathway. PLoS Genet 2:e167

Ferris PJ, Goodenough UW (1994) The mating-type locus of Chlamydomonas reinhardtii contains highly rearranged DNA sequences. Cell 76:1135-1145

Ferris PJ, Goodenough UW (1997) Mating type in Chlamydomonas is specified by Mid, the minusdominance gene. Genetics 146:859-869

Ferris PJ, Pavlovic G, Fabry S et al (1997) Rapid evolution of sex-related genes in Chlamydomonas. Proc Natl Acad Sci USA 94:8634-8639

Ferris P, Olson BJSC, De Hoff PL et al (2010) Evolution of an expanded sex-determining locus in Volvox. Science 328:351-354

Goldstein M (1964) Speciation and mating behavior in Eudorina. J Protozool 11:317-344

Goodenough UW, Detmers PA, Hwang C (1982) Activation for cell fusion in Chlamydomonas: analysis of wild-type gametes and nonfusing mutants. J Cell Biol 92:378-386

Hamaji H, Smith DR, Noguchi H et al (2013) Mitochondrial and plastid genomes of the colonial green alga Gonium pectorale give insights into the origins of organelle DNA architecture within the Volvocales. PLoS One 8:e57177

Herron MD, Hackett JD, Aylward FO et al (2009) Triassic origin and early radiation of multicellular volvocine algae. Proc Natl Acad Sci USA 106:3254-3258

Hiraide R, Kawai-Toyooka H, Hamaji T et al (2013) The evolution of male-female sexual dimorphism predates the gender-based divergence of the mating locus gene MAT3/RB. Mol Biol Evol 30:1038-1040

Kirk DK (1998) Volvox: molecular genetic origins of multicellularity and cellular differentiation. Cambridge University Press, Cambridge

Kirk DL (2006) Oogamy: inventing the sexes. Curr Biol 16:R1028-R1030

Laurin-Lemay S, Brinkmann H, Philippe H (2012) Origin of land plants revisited in the light of sequence contamination and missing data. Curr Biol 22:R593-R594. doi:10.1016/j.cub.2012.06.013

Lynch M (2007) The origins of genome architecture. Sinauer, Sunderland 
Merchant SS, Prochnik SE, Vallon O et al (2007) The Chlamydomonas genome reveals the evolution of key animal and plant functions. Science 318:245-250

Mogi Y, Hamaji T, Suzuki M et al (2012) Evidence for tubular mating structures induced in each mating type of heterothallic Gonium pectorale (Volvocales, Chlorophyta). J Phycol 48:670-674

Nozaki H (1983) Sexual reproduction in Eudorina elegans (Chlorophyta, Volvocales). Bot Mag (Tokyo) 96:103-110

Nozaki H (1984) Newly found facets in the asexual and sexual reproduction in Gonium pectorale (Chlorophyta, Volvocales). Jpn J Phycol 32:130-133

Nozaki H (1988) Morphology, sexual reproduction and taxonomy of Volvox carteri f. kawasakiensis f. nov. (Chlorophyta) from Japan. Phycologia 27:209-220

Nozaki H (1996) Morphology and evolution of sexual reproduction in the Volvocaceae (Chlorophyta). J Plant Res 109:353-361

Nozaki H (2003) Origin and evolution of the genera Pleodorina and Volvox (Volvocales). Biologia 58(4):425-431

Nozaki H (2008) A new male-specific gene "OTOKOGI" from Pleodorina starrii (Volvocaceae, Chlorophyta) unveiling an origin of male and female. Biologia 63:772-777

Nozaki H, Ito M (1994) Phylogenetic relationships within the colonial Volvocales (Chlorophyta) inferred from cladistic analysis based on morphological data. J Phycol 30:353-365

Nozaki H, Ito M, Watanabe MM et al (1996) Ultrastructure of the vegetative colonies and systematic position of Basichlamys (Volvocales, Chlorophyta). Eur J Phycol 31:67-72

Nozaki H, Misawa K, Kajita T et al (2000) Origin and evolution of the colonial Volvocales (Chlorophyceae) as inferred from multiple, chloroplast gene sequences. Mol Phylogenet Evol 17:256-268

Nozaki H, Mori T, Misumi O et al (2006a) Males evolved from the dominant isogametic mating type. Curr Biol 16:R1018-R1020

Nozaki H, Ott FD, Coleman AW (2006b) Morphology, molecular phylogeny and taxonomy of two new species of Pleodorina (Volvoceae, Chlorophyceae). J Phycol 42:1072-1080

Rokas A, Krüger D, Carroll SB (2005) Animal evolution and the molecular signature of radiations compressed in time. Science 310:1933-1938

Smith DR, Lee RW (2009) The mitochondrial and plastid genomes of Volvox carteri: bloated molecules rich in repetitive DNA. BMC Genomics 10:132

Smith DR, Lee RW (2010) Low nucleotide diversity for the expanded organelle and nuclear genomes of Volvox carteri supports the mutational-hazard hypothesis. Mol Biol Evol 27:2244-2256

Smith DR, Hamaji T, Olson BJSC et al (2013) Organelle genome complexity scales positively with organism size in volvocine green algae. Mol Biol Evol 30(4):793-797

Umen JG (2011) Evolution of sex and mating loci: an expanded view from volvocine algae. Curr Opin Microbiol 14:634-641

Umen JG, Goodenough UW (2001) Control of cell division by a retinoblastoma protein homolog in Chlamydomonas. Genes Dev 15:1652-1661 